\title{
THE POTENTIAL OF THE WIDE FIELD CAMERA ON ROSAT FOR INVESTIGATIONS OF THE XUV BACKGROUND
}

\author{
Alan W. Harris and Helen J. Walker \\ Rutherford Appleton Laboratory \\ Chilton, Oxfordshire OX11 0QX, UK
}

\author{
Timothy J. Sumner \\ Imperial College of Science, \\ Technology, and Medicine, \\ London SW7 2BZ, UK
}

The ROSAT X-ray astronomy satellite, due to be launched in early 1990 , will carry two separate and complementary grazing-incidence telescopes with co-aligned axes. The German $X$-ray telescope (XRT) will cover the soft X-ray region in the range $0.15-2 \mathrm{keV}(6-80 \AA)$, while the U.K. XUV Wide Field Camera (WFC) will extend coverage to beyond $200 \AA$. The WFC is a joint project of Leicester and Birmingham Universities, the Mullard Space Science Laboratory, and the authors' institutes. The primary objective of ROSAT is to perform an allsky survey over a period of six months. This will be followed by a guest-observer, "pointed" phase. We briefly discuss the sensitivity of the WFC to the soft X-ray/XUV background (SXRB) and the problems and techniques associated with distinguishing the astronomical background from other sources of background.

The WFC has an unobscured geometrical collecting area of $456 \mathrm{~cm}^{2}$ which focuses radiation onto one of two identical curved microchannel plate (MCP) detectors. The resolution is $2^{\prime}$ FWHM averaged over a $5^{\circ}$ field of view. XUV filters mounted on a wheel in front of the detectors provide continuous wavelength coverage from 60 to $220 \AA$, and a further band around $600 \AA$ (Table 1). The survey will be carried out in two bands, S1 and S2, with a field of view of $5^{\circ}$. Further details of the WFC instrument can be found in Pye (1986) and Barstow and Willingale (1988).

TABLE 1. WFC XUV Bands and Predicted Background Count Rates

\begin{tabular}{lccccc}
\hline \hline \multicolumn{1}{c}{ Filter } & $\begin{array}{c}\text { FOV } \\
(\text { deg. })\end{array}$ & $\begin{array}{c}\text { Bandpass } \\
(\AA)\end{array}$ & $\begin{array}{c}\text { Geocoronal } \\
\left(\text { cts s }^{-1}\right)\end{array}$ & $\begin{array}{c}\text { Particle } \\
\left(\text { cts s }^{-1}\right)\end{array}$ & $\begin{array}{c}\text { Mean SXRB } \\
\left(\text { cts s }^{-1}\right)\end{array}$ \\
\hline S1 (C/Lexan/B) & 5 & $60-140$ & 0 & $10-20$ & 15 \\
S2 (Be/Lexan) & 5 & $15-180$ & $0.5-20$ & $10-20$ & 25 \\
P1 (A1/Lexan) & 2.5 & $150-220$ & $0.5-15$ & $3-6$ & 2 \\
P2 (Sn/Al) ${ }^{1}$ & 2.5 & $540-720$ & $>2000$ & $3-6$ & - \\
\hline
\end{tabular}

${ }^{1}$ This filter is unlikely to be useful for SXRB studies due to the large geocoronal contribution.

Detailed discussions of current speculation on the SXRB and its origins are given by Jakobsen (1986) and Cox and Reynolds (1987). The form of the SXRB energy distribution in the range $0.05-0.2 \mathrm{keV}(-60-250 \AA)$ is well fitted by a power law: photon flux $\left(\mathrm{cm}^{-2} \mathrm{~s}^{-1} \mathrm{sr}^{-1} \mathrm{keV}^{-1}\right)=1.2 \times \mathrm{E}_{\mathrm{keV}^{-3.4}}$ (cf. results compiled by Stern and Bowyer 1979). This represents mean intensity and takes no account of the observed factor $\sim 3$ variation between the galactic equator and poles. Integrating the product of SXRB photon flux and WFC efficiency over the two survey bands gives count rates of 15 and $25 \mathrm{cts} \mathrm{s}^{-1}$ in bands S1 and S2, respectively, in the $5^{\circ}$ field of view.

Unfortunately, the SXRB signal will be contaminated by other sources of background (Table 1). In the S2 band, the geocoronal He II line at $304 \AA$ will give count rates of between 
-0.5 and $20 \mathrm{cts} \mathrm{s}^{-1}$. Since the rate depends on the solar and viewing directions and is predictable, the impact of geocoronal contamination can be minimized by appropriate data selection. The most serious source of background contamination in the WFC may be particle radiation, especially low energy electrons, which will be a function of orbital position. Modeling of low energy electron propagation through the WFC telescope (Sumner et al. 1989) suggests the particle background component will be of the order $10-20 \mathrm{cts} \mathrm{s}^{-1}$ in the survey filters. The WFC filter complement includes a filter which is opaque to XUV photons but mimics the XUV filters in terms of particle transmission, thereby enabling photon and particle radiation to be distinguished. The size of this "opaque" filter corresponds to a field of view of $2.5^{\circ}$. The particle component can be monitored by switching frequently between an XUV filter and the opaque filter. If an XUV $\left(5^{\circ}\right)$ filter is selected for $t \mathrm{~s}$ and the opaque filter for $f \times t \mathrm{~s}$, an estimate of the signal-to-noise ratio for the net SXRB counts in each of $n$ resolution elements in the field of view is given by

$$
S / N \approx C_{\mathrm{sxbb}} t^{1 / 2}\left[n C_{\mathrm{sxrb}}+(n+4 / f) C_{\mathrm{part}}+(n+1 / f) C_{\mathrm{det}}\right]^{-1 / 2},
$$

where $C_{\mathrm{sxrb}}, C_{\text {part }}$, and $C_{\mathrm{det}}$ are the SXRB, particle, and intrinsic detector background cts $\mathrm{s}^{-1}$, respectively, for the $5^{\circ}$ field. Assuming realistic average values for these parameters, i.e., $C_{\text {sxrb }}=20, C_{\text {part }}=15, C_{\text {det }}=10$ and taking $f=1 / 2$ and $t=80 \mathrm{~s}$ (about the maximum time taken for a point on the sky to cross the $5^{\circ}$ field of view in a single survey pass), $5 \sigma$ detection is achieved for $n=25$, i.e., $\sim 1.0^{\circ}$ resolution. During the survey there will be at least 80 passes over a point on the sky, the actual number depending on its ecliptic latitude (survey coverage increases towards the ecliptic holes). It follows that use of the opaque filter in the "switching mode" for just a small fraction of the total survey time would enable the SXRB to be mapped over a substantial part of the sky with resolution of $\leq 1^{\circ}$. The survey is still under consideration. Of course, far better sensitivity and/or resolution will be achievable in the pointed phase when selected fields may be observed for thousands of seconds.

Much can be learned about the location and distribution of the hot gas responsible for the SXRB by comparing spatial variations in the emission with variations in $N(H)$ (e.g., McCammon et al. 1983). However, there are two major shortcomings in the data available to date on the SXRB. First, the resolution of $5-15^{\circ}$ imposed by the short duration of rocket flights, combined with the need to survey as much of the sky as possible, is too coarse to reveal small-scale structure in the SXRB (cf. Bochkarev 1987; Walker, Harris, and Sumner, this volume). Second, data are lacking in the XUV for wavelengths longer than $100 \AA$ at which hydrogen has greater opacity. If the SXRB arises predominantly in the local ISM, then any foreground hydrogen is likely to have a column density of $\leq 1 \times 10^{19} \mathrm{~cm}^{-2}$ and observations at wavelengths longer than $100 \AA$ would be required to reveal absorption effects. Therefore, with an instrumental resolution of $\sim 2^{\prime}$ in a $5^{\circ}$ field and continuous wavelength coverage over the range $60-200 \AA$, the WFC is well equipped to provide vital new data on the SXRB.

\section{REFERENCES}

Barstow, M. A. and Willingale, R. 1988, J. Brit. Interplan. Soc., 41, 337.

Bochkarev, N. G. 1987, Astrophys. Sp. Sci., 138, 229.

Cox, D. P. and Reynolds, R. J. 1987, Ann. Rev. Astron. Astrophys., 25, 303.

Jakobsen, P. 1986, in Proc. COSPAR Symp. on UV Space Astronomy, eds. C. Gry and W. Wamsteker, Advances in Space Research, 6, No. 2, 59.

McCammon, D. et al. 1983, Ap. J., 269, 107.

Pye, J. P. 1986, in Proc. COSPAR Symp. on UV Space Astronomy, eds. C. Gry and W. Wamsteker, Advances in Space Research, 6, No. 2, 139.

Stern, R. and Bowyer, S. 1979, Ap. J., 230, 755.

Sumner, T. J. et al. 1989, Ap. J., 238, 1047.

Walker, H. J., Harris, A. W., and Sumner, T. J. 1989, Proc. IAU 139, Galactic and Extragalactic Background Radiation, ed. S. Bowyer and Ch. Leinert (Dordrecht: Kluwer Academic Publishers). 